\title{
A new salamander from the late Paleocene-early Eocene of Ukraine
}

\author{
Pavel P. Skutschas and Yuri M. Gubin
}

Acta Palaeontologica Polonica 57 (1), 2012: 135-148 doi: http://dx.doi.org/10.4202/app.2010.0101

A new neotenic salamander, Seminobatrachus boltyschkensis gen. et sp. nov., is described based on 14 skeletons of late Paleocene-early Eocene age preserved on drill core slabs from the Cherkassy Region, central Ukraine. The new taxon is diagnosed by the following unique combination of characters: dorsal process of premaxilla posteriorly elongate and overlaps frontal; maxilla greatly reduced in size; parietal-squamosal contact absent; vomerine tooth row long and parallel to maxillary arcade; pterygoid has long anterior process; quadrate ossified; marginal and palatal teeth pedicellate; trunk vertebrae amphicoelous, each having a subcentral keel, anterior basapophysis, and spinal nerve foramina; ribs bicipital; carpals and tarsals unossified; and phalangeal formulae of 2-2-3-2 and 2-2-3-4-2 for manus and pes, respectively. Phylogenetic analysis nests $S$. boltyschkensis within Urodela (i.e., crown-clade salamanders), but its exact phylogenetic position is equivocal, resolving in one of three ways: (1) in an unresolved trichotomy with Salamandra and (Ambystomatidae $+($ Dicamptodon + Rhyacotriton $)$ ) (results obtained in NONAv. 2.0, with the WINCLADAv. 1.00.08 interface; the parsimony ratchet (island hopper) algorithm), (2) as a sister taxon of (Salamandra + (Ambystomatidae $+($ Dicamptodon + Rhyacotriton $))$ ) clade (results obtained in TNT v. 1.1; the implicit enumeration search algorithm) or (3) as a sister taxon of Ambystomatidae (results obtained in PAUP v. 4.0b10; the branch-and-bound search algorithm).

Key words: Caudata, Urodela, phylogeny, Paleocene, Eocene, Ukraine.

Pavel P. Skutschas [kutchas@mail.ru], Saint Petersburg State University, Vertebrate Zoology Department, Faculty of Biological and Soil Sciences, Universitetskaya nab. 7/9, Saint Petersburg 199034, Russian Federation; Yuri M. Gubin [gubin@paleo.ru], Paleontological Institute, Russian Academy of Sciences, Profsoyuznaya ul. 123, Moscow 117647, Russian Federation.

This is an open-access article distributed under the terms of the Creative Commons Attribution License (for details please see creativecommons.org), which permits unrestricted use, distribution, and reproduction in any medium, provided the original author and source are credited. 
PaF Full text $(1,241.1 \mathrm{kB})$ 